\title{
VALORACIÓN FUNCIONAL DE PACIENTES CON LESIONES DEL MIEMBRO SUPERIOR A TRA-
} VÉS DEL CUESTIONARIO DASHe

\section{FUNCTIONAL ASSESSMENT OF PATIENTS WITH INJURIES OF THE HIGHER MEMBER THROUGH THE DASHe QUESTIONNAIRE}

\author{
Marcos ALMIRÓN¹, Iván BARRIOS². \\ ${ }^{1}$ Kinesiólogo, Facultad de Ciencias Médicas, Universidad Nacional de Asunción, San Lorenzo - Para- \\ guay. \\ ${ }^{2}$ Estudiante de Medicina y Cirugía, Facultad de Ciencias Médicas, Universidad Nacional de Asunción, \\ San Lorenzo - Paraguay.
}

Cómo citar este artículo: Almirón M, Barrios I. Valoración funcional de pacientes con lesiones de miembro superior a través del cuestionario DASHe. Medicina Clínica y Social. 2018;2(3):136-144.

\section{RESUMEN}

Introducción: La valoración funcional es esencial en el manejo de los problemas del miembro superior, tanto para la toma de decisiones diagnósticas y terapéuticas como para evaluar la evolución y la efectividad de los tratamientos instaurados. Objetivo: sido valorar la funcionalidad de pacientes con lesiones del miembro superior, pre y post tratamiento. Metodología: estudio observacional, longitudinal, analítico de prevalencia. No probabilístico, a criterio. La población accesible fueron pacientes adultos con lesiones del miembro superior que son tratados en el Servicio de Kinesiología y Fisioterapia entre julio a octubre de 2018. Resultados: 20 pacientes fueron incluidos en la investigación de los cuales el 55\% es del sexo masculino. Al inicio de la investigación la puntuación DASHe fue de un minino de 6,7 a 73,6 puntos y una media de $33 \pm 22,1$ puntos, después del tratamiento aplicado los puntajes oscilaron entre 1,7 y 55 con una media de 17,8 \pm 14,6 puntos. Discusión: Como se ha visto la consistencia interna de la adaptación al español es buena, puesto que el alfa de Cronbach indica una muy buena validez. La disminución en la escala DASHe fue estadísticamente significativa.

Palabras clave: Valoración funcional; Kinesiología; Miembro superior.

\section{ABSTRACT}

Introduction: Functional assessment is essential in the management of the problems of the upper limb, both for the taking of diagnostic and therapeutic decisions and for evaluating the evolution and effectiveness of the treatments instituted. Objective: to assess the functionality of patients with lesions of the upper limb, pre- and posttreatment. Methodology: observational, longitudinal, analytical prevalence study. Not probabilistic, to criterion. The accessible population were adult patients with lesions of the upper limb who are treated in the Kinesiology and Physiotherapy Service between July to October 2018. Results: 20 patients were included in the research of which $55 \%$ is male. At the beginning of the investigation, the DASH score ranged from 6.7 to 73.6 points and an average of $33 \pm 22.1$ points, after the applied treatment the scores ranged between 1.7 and 55 with an average of $17.8 \pm 14.6$ points. Discussion: As we have seen, the internal consistency of the adaptation to Spanish is good, since Cronbach's alpha indicates a very good validity. The decrease in the DASHe scale was statistically significant.

Keywords: Functional assessment; Kinesiology; Upper limb. 


\section{INTRODUCCIÓN}

La valoración funcional es esencial en el manejo de los problemas del miembro superior, tanto para la toma de decisiones diagnósticas y terapéuticas como para evaluar la evolución y la efectividad de los tratamientos instaurados. Esta valoración puede realizarse mediante algunas exploraciones y escalas funcionales que permiten objetivar el impacto de la enfermedad sobre las estructuras y la función del miembro superior, pero, dada la importancia de la perspectiva subjetiva del propio paciente, especialmente en aspectos como el alivio de los síntomas y la reducción de la discapacidad para sus actividades habituales, existe un creciente interés por la búsqueda de medidas de calidad de vida relacionada con la salud (CVRS) específicas para los problemas de estas extremidades (1).

Existen diversas escalas que valoran la CVRS en problemas del miembro superior, pero en su mayor parte están enfocadas a una articulación o región anatómica (hombro, codo o mano) o a una enfermedad concreta (p. ej., síndrome del túnel carpiano). Aunque estas escalas son útiles, es conocida la importante interrelación entre las diferentes regiones del miembro superior, donde la función en una de ellas afecta al resto de regiones (2), y, por otro lado, en muchos casos se requiere comparar grupos de pacientes diferentes, para lo que se precisa una escala común.

El cuestionario Disabilities of Arm, Shoulder and Hand (DASH) se diseñó para superar estas limitaciones (3). El DASH original es un cuestionario autoadministrado, que valora el miembro superior como una unidad funcional y permite cuantificar y comparar la repercusión de los diferentes procesos que afectan a distintas regiones de dicha extremidad. Desarrollado a iniciativa de la American Academy of Orthopedic Surgeons, se ha utilizado en numerosos trabajos (tanto en rehabilitación y reumatología como en cirugía ortopédica y traumatología), su fiabilidad, validez y sensibilidad a los cambios son bien conocidas (3-15), y en EE UU dispone de valores normativos poblacionales (16). El DASH se ha traducido a diversos idiomas y se han realizado adaptaciones transculturales al sueco $(17,18)$, francés $(19,20)$, alemán $(21-24)$, italiano (25), chino cantonés $(26,27)$, portugués de Brasil (28), armenio (29), ruso (29), japonés (30) y al español $(1,31)$.

En el caso específico de la versión española, el uso de este cuestionario en debe ser adaptado para las diferentes tonalidades del español habladas en España y América Latina, por lo que se consideró importante realizar una adaptación cultural al español de Paraguay del cuestionario DASH, verificando su fiabilidad y validez.

En ese sentido, el objetivo ha sido valorar la funcionalidad de pacientes con lesiones del miembro superior, pre y post tratamiento, analizando la fiabilidad y validez de una versión adaptada al español paraguayo del cuestionario DASHe de Hervás y colaboradores.

\section{METODOLOGÍA}

Estudio observacional, longitudinal, analítico de prevalencia. No probabilístico, a criterio. La población accesible fueron pacientes adultos con lesiones del miembro superior que son tratados en el Servicio de Kinesiología y Fisioterapia de la Facultad de Ciencias Médicas de la 
Universidad Nacional de Asunción entre julio a octubre de 2018. Se incluyó a pacientes mayores a 18 años de ambos sexos, pacientes con lesiones del miembro superior, con o sin tratamiento médico/quirúrgico previo, pacientes sin tratamiento fisioterapéutico previo de la zona anatómica del tren superior por la cual consultan, pacientes que sepan leer. Se excluirá a pacientes con tratamiento fisioterapéutico previo de la zona anatómica del tren superior por la cual consultan y pacientes que se nieguen a participar de la investigación.

Para la adaptación al español paraguayo del Cuestionario DASHe

Sobre la versión en español del cuestionario DASHe, de Hervás y colaboradores, se realizaron adaptaciones culturales al español paraguayo. Los cambios en el cuestionario fueron validados por al menos cuatro expertos en Kinesiología y Fisioterapia, que tengan el español como lengua materna. Luego se realizó un pre-test en cinco usuarios, quiénes no fueron incluidos en la investigación final.

Para la valoración funcional de los pacientes

Previa autorización de las Jefaturas de los Departamentos de Lesiones Músculo-Esqueléticas del Miembro Superior y Columna y de Rehabilitación Traumato-Ortopédica, se incluyó en el estudio a cada paciente que consulte al Servicio de Kinesiología y Fisioterapia y que cumpla con los criterios de inclusión.

Los pacientes fueron invitados a participar del estudio, previa firma de un consentimiento informado. Una vez realizado esto, antes de iniciar la primera sesión fisioterapéutica (según el diagnóstico del paciente), el paciente completó el cuestionario DASHe. Finalmente, al alta del tratamiento fisioterapéutico, se solicitó al paciente que vuelva a rellenar el cuestionario.

Las variables fueron: sexo (hombre mujer), edad (en años cumplidos), nivel de estudios, estado civil, situación laboral, comorbilidad, región anatómica, lado afecto, diagnóstico, tipo de lesión, y resultado del cuestionario DASHe. EI DASH permite valorar la discapacidad percibida por el paciente para realizar diversas actividades, incluidas actividades de la vida diaria y síntomas como el dolor, la rigidez o la pérdida de fuerza.

EI DASH es un cuestionario autoadministrado, que consta de un cuerpo central (que mide discapacidad/síntoma) de 30 ítems y 2 módulos opcionales, con 4 ítems cada uno (32). Cada ítem se puntúa de 1 a 5 , con valores crecientes en función de la intensidad de los síntomas. En el caso de las preguntas de discapacidad/síntomas (30 preguntas, puntuación del 1-5), para poder calcular la puntuación hay que completar al menos 27 de las 30 preguntas. Se suman los valores asignados a cada una de las respuestas completadas y se halla el promedio, obteniendo así una puntuación del uno al cinco. Para expresar esta puntuación en por cientos, se le resta 1 y se multiplica por 25. A mayor puntuación, mayor discapacidad. Los módulos opcionales, en su caso, se puntúan por separado siguiendo el mismo método.

Si la persona deja sin contestar más del 10 por ciento de las preguntas (es decir, más de 3 preguntas), no se podrá calcular la puntuación DASH de discapacidad/síntoma. Siguiendo esta 
misma regla (es decir, no se pueden dejar sin contestar más del 10 por ciento de las preguntas), no es aceptable que se dejen preguntas sin contestar en las secciones opcionales de trabajo/ocupación y de atletas de alto rendimiento/músicos, porque cada sección consta solamente de 4 preguntas. Al ser este el primer estudio de valoración funcional de pacientes con lesiones del miembro superior a través del cuestionario DASHe, en el que además se ha realizado una adaptación cultural al español de Paraguay, se considera el presente como un estudio piloto.

Se realizó en primer lugar un análisis descriptivo de las características sociodemográficas y clínicas de la muestra, así como de las puntuaciones obtenidas en los cuestionarios DASHe adaptado al español de Paraguay. Se comparan las medias de las puntuaciones obtenidas por los pacientes antes y después del tratamiento fisioterapéutico a través de la prueba $t$ para muestras relacionadas. En segundo lugar, se valoró la fiabilidad del cuestionario mediante el análisis de consistencia interna, para lo que se utilizó el coeficiente alfa de Cronbach. Los análisis se realizaron utilizando el programa estadístico Epi Info, versión libre, 7.2 (CDC, Estados Unidos de América).

Esta investigación no representa una intervención, por lo que no se plantearan los dilemas éticos de esa perspectiva. Se cumplirán con los siguientes principios de autonomía, beneficencia y no maleficencia y de justicia.

\section{RESULTADOS}

20 pacientes fueron incluidos en la investigación de los cuales el $55 \%$ es del sexo masculino con edades comprendidas entre los 18 y los 65 años y una edad promedio de $46 \pm 14$ años, en cuanto al nivel de estudios, el $35 \%$ tiene la secundaria completa, el $50 \%$ es casado, el $65 \%$ no trabaja, el $10 \%$ presenta alguna comorbilidad, mano y muñeca fueron las regiones anatómicas más afectadas con el $40 \%$.

El 70\% de las lesiones fue en el lado dominante y el principal diagnóstico fue una indicación posterior a una cirugía en el $35 \%$ de los casos, el $65 \%$ de las lesiones fue en partes blandas. EI resto de estas características clínico - epidemiológicas se observa en la Tabla 1.

\begin{tabular}{|l|c|c|}
\hline \multicolumn{2}{|c|}{ TABLA 1. CARACTERÍSTICAS CLÍNICO - EPIDEMIOLÓGICAS DE LOS PACIENTES (N=20) } \\
\hline \multicolumn{1}{|c|}{ Variable } & Frecuencia & Porcentaje \\
\hline Sexo & 11 & 55 \\
Hombre & 9 & 45 \\
Mujer & 6 & 30 \\
Nivel de estudios & 1 & 5 \\
Primaria incompleta & 4 & 20 \\
Primaria completa & 7 & 35 \\
Secundaria incompleta & 2 & 10 \\
Secundaria completa & & 30 \\
Terciaria completa & 6 & 50 \\
Estado Civil & 10 & 15 \\
Soltero & 3 & \\
Casado & & \\
Pareja de hecho & & \\
\end{tabular}




\begin{tabular}{|c|c|c|}
\hline Viudo & 1 & 5 \\
\hline \multicolumn{3}{|l|}{ Situación Laboral } \\
\hline Trabaja & 7 & 35 \\
\hline No trabaja & 13 & 65 \\
\hline \multicolumn{3}{|l|}{ Comorbilidad } \\
\hline Sí & 2 & 10 \\
\hline No & 18 & 90 \\
\hline \multicolumn{3}{|l|}{ Región anatómica } \\
\hline Hombro & 7 & 35 \\
\hline Brazo & 1 & 5 \\
\hline Codo & 4 & 20 \\
\hline Mano/muñeca & 8 & 40 \\
\hline \multicolumn{3}{|l|}{ Lado afecto } \\
\hline Dominante & 14 & 70 \\
\hline No dominante & 6 & 30 \\
\hline \multicolumn{3}{|l|}{ Diagnóstico } \\
\hline Post cirugía & 7 & 35 \\
\hline Tendinitis & 1 & 5 \\
\hline Lesión de Bankart & 1 & 5 \\
\hline Lesión nervio radial & 1 & 5 \\
\hline Artritis rematoidea & 2 & 10 \\
\hline Lesión de partes blandas & 4 & 20 \\
\hline Luxación & 1 & 5 \\
\hline Fractura & 2 & 10 \\
\hline Epicondilitis & 1 & 5 \\
\hline \multicolumn{3}{|l|}{ Tipo de lesión } \\
\hline Fractura/fisura & 7 & 35 \\
\hline Partes blandas & 13 & 65 \\
\hline \multicolumn{3}{|l|}{ Tratamiento } \\
\hline Quirúrgico & 12 & 60 \\
\hline Conservador & 8 & 40 \\
\hline
\end{tabular}

En cuanto a la fiabilidad del DASHe en su versión para el español paraguayo, vemos que antes del tratamiento tiene un alfa de Cronbach de 0,943 y después del tratamiento de 0,944 lo que indica una buena consistencia del instrumento y una buena concordancia antes y después del tratamiento aplicado a los participantes.

Al inicio de la investigación la puntuación DASHe fue de un minino de 6,7 a 73,6 puntos y una media de $33 \pm 22,1$ puntos, después del tratamiento aplicado los puntajes oscilaron entre 1,7 y 55 con una media de 17,8 $\pm 14,6$ puntos. Esta diferencia es estadísticamente significativa $(p=0,001)$ por lo que el tratamiento aplicado redujo la puntuación DASHe, por lo tanto, aumentó la funcionalidad. La correlación entre grupos fue de 0,631 y significativo $(p=0,003)$.

\section{DISCUSIÓN}

Como se ha visto la consistencia interna de la adaptación al español es buena, puesto que el alfa de Cronbach indica una muy buena validez. Los pacientes incluidos en nuestra muestra fueron principalmente hombres, esto está en concordancia con otras investigaciones similares donde también hubo más hombres que mujeres en los estudios que involucran la escala DASH (32) debería investigarse si dicha diferencia se debe a aceptar participar de la investigación o a que las lesiones de miembro superior son más frecuentes en los hombres. 
En cuanto a la comorbilidad, en esta investigación la frecuencia encontrada fue de $10 \%$, sin embargo, en la literatura se reportan frecuencias de $34 \%$, donde las principales comorbilidades son: enfermedad renal crónica, diabetes, enfermedad intestinal inflamatoria, enfermedad autoinmune, cáncer, etc. (32). Esta investigación se limitó a buscar la presencia o ausencia de comorbilidades, podría usarse la frecuencia encontrada para en próximos estudios indagar sobre las principales comorbilidades en la población enfocada de la presente investigación.

Una de las medidas de resultado informadas por el paciente es el cuestionario de discapacidad del brazo, hombro y mano (DASH), que es una medida de resultado específica de la extremidad superior. La razón detrás del uso de una medida de resultado para diferentes trastornos de las extremidades superiores es que las extremidades superiores son una unidad funcional. Es por ello que este cuestionario es de fundamental importancia dada su practicidad en la consulta y debería ser utilizado en forma rutinaria en los servicios de fisioterapia antes del tratamiento para un mejor seguimiento de los pacientes y así tener un control "cuantitativo" del progreso del paciente al alta.

La puntuación DASHe antes del tratamiento fue de $33 \pm 22,1$ muy por encima del 13,3 $\pm 12,3$ reportado por otros autores (33), esto debe entenderse desde el punto de vista de que el dolor es un síntoma, por tanto es subjetivo y depende del umbral de dolor de cada persona, así también la funcionalidad o no, es algo subjetivo de cada paciente, por lo cual resuelta más útil comparar los valores de DASHe antes de después de un tratamiento (para que ambas medidas sean tomadas en los mismos individuos) de esta forma más que comparar los puntajes en poblaciones similares es importante comparar los puntajes en los mismos individuos antes de después del tratamiento, ya que es una forma rápida de valorar la funcionalidad del miembro superior.

Luego del tratamiento aplicado el puntaje DASH pasó a ser de 17,8 $\pm 14,6$ lo que implica una caída de 15 puntos aproximadamente como consecuencia del tratamiento ya que al realizar la prueba de significancia con la prueba t para muestras emparejadas se obtuvo un resultado significativo $(p<0,01)$, así mismo la correlación entre los grupos fue positiva e igual a 0,6 $(p<0,01)$ lo que nos da la seguridad de que efectivamente se trataba de dos grupos relacionadas y podía utilizarse esta prueba de contraste de hipótesis.

Ya se ha reportado en la literatura que el DASH es útil para evaluar la efectividad del tratamiento aplicado a los pacientes, también dada su buena correlación es útil como un control evolutivo que puede tomarse antes del tratamiento, y a los tres meses, 6 meses, con el fin de ver el progreso en la funcionalidad $(34,35)$.

Entre las limitaciones de la investigación se encuentra que al ser el primer estudio sobre el tema en la región tuvo que empezar con un diseño piloto lo cual impide utilizar un muestreo de tipo probabilístico, por lo que los resultados presentados solo pueden interpretarse y aplicarse a la muestra estudiada, no así generalizar los datos a toda la población de pacientes que acuden al servicio a recibir este tipo de terapia. Sin embargo, el hecho de que sea un estudio piloto facilitará las próximas investigaciones sobre el tema, puesto que servirá para calcular un adecuado tamaño de muestra y de esta forma realizar también un muestreo probabilístico con el fin de obtener resultados no solo con validez interna, sino también con validez externa. 


\section{CONFLICTOS DE INTERÉS Y FUENTE DE FINANCIACIÓN}

Los autores declaran no poseer conflictos de interés. Fuente de financiación: ninguna.

\section{REFERENCIAS BIBLIOGRÁFICAS}

1. Hervás J, Collado MJN, Moreno SP, Pérez JLR, Matéu PL, Tello IM. Versión española del cuestionario DASH. Adaptación transcultural, fiabilidad, validez y sensibilidad a los cambios. Med Clínica. 2006;127(2):441-7. https://doi.org/10.1157/13093053

2. Eggers IM, Mennen U. The EFFUL (Evaluation of Function in the Flail Upper Limb) system. A ranking score system to measure improvement achieved by surgical reconstruction and rehabilitation. J Hand Surg Edinb Scotl. 1997;22(3):388-94. URL.

3. Hudak PL, Amadio PC, Bombardier C. Development of an upper extremity outcome measure: the DASH (disabilities of the arm, shoulder and hand) [corrected]. The Upper Extremity Collaborative Group (UECG). Am J Ind Med. 1996;29(6):602-8. https://doi.org/10.1002/(SICI)1097-0274(199606)29:6\%3C602::AIDAJIM4\%3E3.0.CO;2-L

4. Turchin DC, Beaton DE, Richards RR. Validity of observer-based aggregate scoring systems as descriptors of elbow pain, function, and disability. J Bone Joint Surg Am. 1998;80(2):154-62. http://dx.doi.org/10.2106/00004623-199802000-00002

5. Navsarikar A, Gladman DD, Husted JA, Cook RJ. Validity assessment of the disabilities of arm, shoulder, and hand questionnaire (DASH) for patients with psoriatic arthritis. J Rheumatol. 1999;26(10):2191-4. URL.

6. MacDermid JC, Richards RS, Donner A, Bellamy N, Roth JH. Responsiveness of the short form-36, disability of the arm, shoulder, and hand questionnaire, patient-rated wrist evaluation, and physical impairment measurements in evaluating recovery after a distal radius fracture. J Hand Surg. 2000;25(2):330-40. https://doi.org/10.1053/jhsu.2000.jhsu25a0330

7. Beaton DE, Katz JN, Fossel AH, Wright JG, Tarasuk V, Bombardier C. Measuring the whole or the parts? Validity, reliability, and responsiveness of the Disabilities of the Arm, Shoulder and Hand outcome measure in different regions of the upper extremity. J Hand Ther Off J Am Soc Hand Ther. 2001;14(2):128-46. URL.

8. Jain R, Hudak PL, Bowen CV. Validity of health status measures in patients with ulnar wrist disorders. J Hand Ther Off J Am Soc Hand Ther. 2001;14(2):147-53. URL.

9. SooHoo NF, McDonald AP, Seiler JG, McGillivary GR. Evaluation of the construct validity of the DASH questionnaire by correlation to the SF-36. J Hand Surg. 2002;27(3):537-41. http://dx.doi.org/10.1053/jhsu.2002.32964

10. Gay RE, Amadio PC, Johnson JC. Comparative responsiveness of the disabilities of the arm, shoulder, and hand, the carpal tunnel questionnaire, and the SF-36 to clinical change after carpal tunnel release. J Hand Surg. 2003;28(2):250-4. https://doi.org/10.1053/jhsu.2003.50043

11. MacDermid JC, Tottenham V. Responsiveness of the disability of the arm, shoulder, and hand (DASH) and patient-rated wrist/hand evaluation (PRWHE) in evaluating change after hand therapy. J Hand Ther Off J Am Soc Hand Ther. 2004;17(1):18-23. https://doi.org/10.1197/j.jht.2003.10.003 
12. Greenslade JR, Mehta RL, Belward P, Warwick DJ. Dash and Boston questionnaire assessment of carpal tunnel syndrome outcome: what is the responsiveness of an outcome questionnaire? J Hand Surg Edinb Scotl. 2004;29(2):159-64. https://doi.org/10.1016/j.jhsb.2003.10.010

13. Schmitt JS, Di Fabio RP. Reliable change and minimum important difference (MID) proportions facilitated group responsiveness comparisons using individual threshold criteria. J Clin Epidemiol. 2004;57(10):1008-18. https://doi.org/10.1016/j.jclinepi.2004.02.007

14. Kotsis SV, Chung KC. Responsiveness of the Michigan Hand Outcomes Questionnaire and the Disabilities of the Arm, Shoulder and Hand questionnaire in carpal tunnel surgery. J Hand Surg. 2005;30(1):81-6. https://doi.org/10.1016/j.jhsa.2004.10.006

15. Hobby JL, Watts C, Elliot D. Validity and responsiveness of the patient evaluation measure as an outcome measure for carpal tunnel syndrome. J Hand Surg Edinb Scotl. 2005;30(4):350-4. https://doi.org/10.1016/j.jhsb.2005.03.009

16. Hunsaker FG, Cioffi DA, Amadio PC, Wright JG, Caughlin B. The American academy of orthopaedic surgeons outcomes instruments: normative values from the general population. J Bone Joint Surg Am. 2002;84-A(2):208-15. URL.

17. Atroshi I, Gummesson C, Andersson B, Dahlgren E, Johansson A. The disabilities of the arm, shoulder and hand (DASH) outcome questionnaire: reliability and validity of the Swedish version evaluated in 176 patients. Acta Orthop Scand. 2000;71(6):613-8. https://doi.org/10.1080/000164700317362262

18. Gummesson C, Atroshi I, Ekdahl C. The disabilities of the arm, shoulder and hand (DASH) outcome questionnaire: longitudinal construct validity and measuring selfrated health change after surgery. BMC Musculoskelet Disord. 2003;4:11. https://dx.doi.org/10.1186\%2F1471-2474-4-11

19. Dubert T, Voche P, Dumontier C, Dinh A. Le questionnaire DASH. Adaptation française d'un outil d'évaluation international. /data/revues/12973203/v0020i04/0100049x/. 2001;20(4):294-302. URL.

20. Durand M-J, Vachon B, Hong QN, Loisel P. The cross-cultural adaptation of the DASH questionnaire in Canadian French. $\mathbf{J}$ Hand Ther Off $\mathbf{J}$ Am Soc Hand Ther. 2005;18(1):34-9. https://doi.org/10.1197/j.jht.2004.10.010

21. Veehof MM, Sleegers EJA, van Veldhoven NHMJ, Schuurman AH, van Meeteren NLU. Psychometric qualities of the Dutch language version of the Disabilities of the Arm, Shoulder, and Hand questionnaire (DASH-DLV). J Hand Ther Off J Am Soc Hand Ther. 2002;15(4):347-54. http://dx.doi.org/10.1016/S0894-1130(02)80006-0

22. De Smet L. Responsiveness of the DASH score in surgically treated basal joint arthritis of the thumb: preliminary results. Clin Rheumatol. 2004;23(3):223-4. https://doi.org/10.1007/s10067-004-0866-y

23. Offenbächer M, Ewert T, Sangha O, Stucki G. Validation of a German version of the «Disabilities of Arm, Shoulder and Hand» questionnaire (DASH-G). Z Rheumatol. 2003;62(2):168-77. https://doi.org/10.1007/s00393-003-0461-7

24. Germann G, Harth A, Wind G, Demir E. [Standardisation and validation of the German version 2.0 of the Disability of Arm, Shoulder,Hand (DASH) questionnaire]. Unfallchirurg. 2003;106(1):13-9. https://doi.org/10.1007/s00113-002-0456-X

25. Padua R, Padua L, Ceccarelli E, Romanini E, Zanoli G, Amadio PC, et al. Italian version of the Disability of the Arm, Shoulder and Hand (DASH) questionnaire. Cross-cultural adaptation and validation. J Hand Surg Edinb Scotl. 2003;28(2):179-86. http://dx.doi.org/10.1016/S0266-7681(02)00303-0 
26. Lee EWC, Lau JSY, Chung MMH, Li APS, Lo SK. Evaluation of the Chinese version of the Disability of the Arm, Shoulder and Hand (DASH-HKPWH): cross-cultural adaptation process, internal consistency and reliability study. J Hand Ther Off J Am Soc Hand Ther. 2004;17(4):417-23. http://dx.doi.org/10.1197/j.jht.2004.07.005

27. Lee EWC, Chung MMH, Li APS, Lo SK. Construct validity of the Chinese version of the disabilities of the arm, shoulder and hand questionnaire (DASH-HKPWH). J Hand Surg Edinb Scotl. 2005;30(1):29-34. https://doi.org/10.1016/j.jhsb.2004.09.010

28. Orfale AG, Araújo PMP, Ferraz MB, Natour J. Translation into Brazilian Portuguese, cultural adaptation and evaluation of the reliability of the Disabilities of the Arm, Shoulder and Hand Questionnaire. Braz J Med Biol Res Rev Bras Pesqui Medicas E Biol. 2005;38(2):293-302. https://doi.org//S0100-879X2005000200018

29. Gevorgyan A, Abrahamyan DO, Yaghjyan GV. Translation and Cross-Cultural Adaptation of the Eastern Armenian and Russian Versions of the Disabilities of the Arm, Shoulder, and Hand Outcome Measure. J Hand Surg. 2006;31(9):1550-1. https://doi.org/10.1016/j.jhsa.2006.08.006

30. Imaeda T, Toh S, Nakao Y, Nishida J, Hirata H, Ijichi M, et al. Validation of the Japanese Society for Surgery of the Hand version of the Disability of the Arm, Shoulder, and Hand questionnaire. J Orthop Sci Off J Jpn Orthop Assoc. 2005;10(4):353-9. https://doi.org/10.1007/s00776-005-0917-5

31. Rosales RS, Delgado EB, Díez de la Lastra-Bosch I. Evaluation of the Spanish version of the DASH and carpal tunnel syndrome health-related quality-of-life instruments: cross-cultural adaptation process and reliability. J Hand Surg. 2002;27(2):334-43. http://dx.doi.org/10.1053/jhsu.2002.30059

32. Tosetto A, Testa S, Martinelli I, Poli D, Cosmi B, Lodigiani C, et al. External validation of the DASH prediction rule: a retrospective cohort study. J Thromb Haemost. 2017;15(10):1963-70. https://doi.org/10.1111/jth.13781

33. Izawa KP, Kasahara Y, Hiraki K, Hirano Y, Watanabe S. Relation between the Disability of the Arm, Shoulder and Hand Score and Muscle Strength in Post-Cardiac Surgery Patients. Diseases. 2017;5(4):31. https://dx.doi.org/10.3390\%2Fdiseases5040031

34. Björnsson Hallgren HC, Adolfsson LE, Johansson K, Öberg B, Peterson A, Holmgren TM. Specific exercises for subacromial pain. Acta Orthop. 2017;88(6):600-5. https://dx.doi.org/10.1080\%2F17453674.2017.1364069

35. Sayegh ET, Strauch RJ. Does Nonsurgical Treatment Improve Longitudinal Outcomes of Lateral Epicondylitis Over No Treatment? A Meta-analysis. Clin Orthop. 2015;473(3):1093-107. https://dx.doi.org/10.1007\%2Fs11999-014-4022-y 